EXTENDED REPORT

\title{
Efficacy of physiotherapy management of knee joint osteoarthritis: a randomised, double blind, placebo controlled trial
}

\author{
K L Bennell, R S Hinman, B R Metcalf, R Buchbinder, J McConnell, G McColl, S Green, K M Crossley
}

Ann Rheum Dis 2005;64:906-912. doi: 10.1136/ard.2004.026526

See end of article for authors' affiliations

Correspondence to:

Associate

Professor Kim Bennell,

Centre for Health, Exercise

and Sports Medicine,

School of Physiotherapy,

University of Melbourne,

Parkville, Victoria,

Australia 3010;

k.bennell@unimelb.edu.au

Accepted

1 November 2004

\begin{abstract}
Objective: To determine whether a multimodal physiotherapy programme including taping, exercises, and massage is effective for knee osteoarthritis, and if benefits can be maintained with self management. Methods: Randomised, double blind, placebo controlled trial; 140 community volunteers with knee osteoarthritis participated and 119 completed the trial. Physiotherapy and placebo interventions were applied by 10 physiotherapists in private practices for 12 weeks. Physiotherapy included exercise, massage, taping, and mobilisation, followed by 12 weeks of self management. Placebo was sham ultrasound and light application of a non-therapeutic gel, followed by no treatment. Primary outcomes were pain measured by visual analogue scale and patient global change. Secondary measures included WOMAC, knee pain scale, SF-36, assessment of quality of life index, quadriceps strength, and balance test. Results: Using an intention to treat analysis, physiotherapy and placebo groups showed similar pain reductions at 12 weeks: $-2.2 \mathrm{~cm}(95 \% \mathrm{Cl},-2.6$ to -1.7$)$ and $-2.0 \mathrm{~cm}(-2.5$ to -1.5$)$, respectively. At 24 weeks, pain remained reduced from baseline in both groups: $-2.1(-2.6$ to -1.6$)$ and $-1.6(-2.2$ to $-1.0)$, respectively. Global improvement was reported by $70 \%$ of physiotherapy participants $(51 / 73)$ at 12 weeks and by $59 \%(43 / 73)$ at 24 weeks. Similarly, global improvement was reported by $72 \%$ of placebo participants $(48 / 67)$ at 12 weeks and by $49 \%(33 / 67)$ at 24 weeks (all $p>0.05)$.

Conclusions: The physiotherapy programme tested in this trial was no more effective than regular contact with a therapist at reducing pain and disability.
\end{abstract}

K nee osteoarthritis is a prevalent musculoskeletal condition affecting older people, ${ }^{1}$ causing pain, physical disability, and reduced quality of life. It also imposes a considerable economic burden on the health care system. ${ }^{2}$ Given that people over the age of 65 will comprise almost 25\% of the US population by $2040,{ }^{3}$ the public health problem of knee osteoarthritis should increase.

Physiotherapy is a non-pharmacological intervention for knee osteoarthritis recommended by the American College of Rheumatology and the European League Against Rheumatism. ${ }^{45}$ It encompasses numerous treatment modes including exercise, manual techniques, knee taping, and education to impart patient self management strategies. Most studies of physiotherapy for knee osteoarthritis have evaluated individual components, but this does not reflect typical clinical practice. While three randomised controlled trials have investigated a physiotherapy treatment package for knee osteoarthritis, ${ }^{6-8}$ only one used a placebo comparison group. ${ }^{7}$ Two trials reported a beneficial effect of physiotherapy, ${ }^{67}$ while one reported no effect. ${ }^{8}$ However, results are difficult to compare owing to different osteoarthritis samples and treatments employed. Given the strong placebo effect reported for pain outcomes in surgical and drug trials in this patient population, ${ }^{910}$ further placebo controlled trials of physiotherapy are clearly needed.

To build the evidence base for non-pharmacological management of knee osteoarthritis, we aimed to test the efficacy of a novel multifaceted physiotherapy programmeincluding functional exercise, massage, and knee taping-on pain and disability compared with a placebo control. We also aimed to determine whether any physiotherapy benefits could be maintained by self management strategies. It was hypothesised that physiotherapy would be superior to a placebo intervention.

\section{METHODS}

\section{Participants}

A community sample was recruited in Melbourne, Australia, through advertisements placed in local media. Selection criteria (based on the clinical and radiological criteria defined by the American College of Rheumatology ${ }^{11}$ for knee osteoarthritis) included age $\geqslant 50$ years, knee pain on most days of the past month (average pain severity of $\geqslant 4$ on an 11 point numerical rating scale), osteophytes on $x$ ray (as assessed by an experienced radiologist), and pain or difficulty in rising from sitting or climbing stairs. Exclusion criteria included physiotherapy or knee surgery (in the previous 12 months), lower limb arthroplasty, Synvisc ${ }^{\circledR}$ or intra-articular steroid injections (in the previous six months), a systemic arthritic condition, a severe medical condition, poor skin condition, known allergic reaction to tape, or a body mass index of more than $36 \mathrm{~kg} / \mathrm{m}^{2}$ (which would result in difficulty in knee taping). All participants were initially screened over the phone with regard to selection criteria and if appropriate underwent medical screening with a project rheumatologist (RB or GM).

The University of Melbourne human research ethics committee approved the study and all participants provided written informed consent.

\section{Protocol}

The trial comprised a 12 week intervention and a 12 week follow up (fig 1). Participants were assessed immediately

Abbreviations: $A Q o L$, assessment of quality of life index; SF-36, short form 36 item general health questionnaire; VAS, visual analogue scale; WOMAC, Western Ontario and McMaster Universities osteoarthritis index 


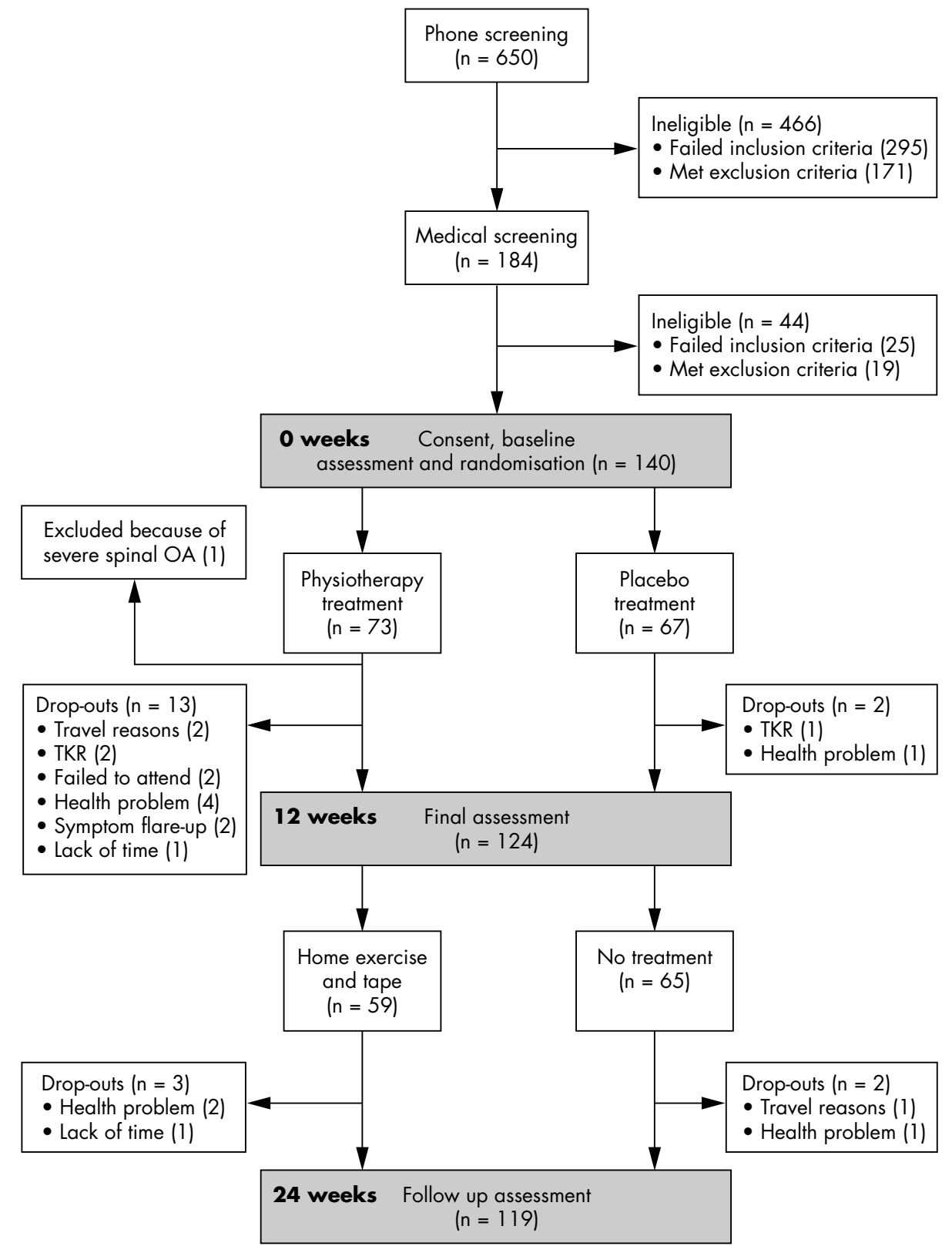

Figure 1 The trial protocol.

before treatment (baseline), immediately after treatment (final), and at 24 weeks (follow up).

\section{Assignment}

Following baseline assessment, participants were randomly assigned to physiotherapy or placebo treatment. Simple randomisation was employed using a computer generated table of random numbers. An independent researcher not involved in eligibility assessment, outcome assessment, or treatment kept the concealed assignment scheme in a locked cupboard in a central location. Allocation was revealed to the treating physiotherapist by telephone at the time the participant presented for treatment.

\section{Interventions}

Ten experienced musculoskeletal physiotherapists employed in private practice were selected to provide geographical coverage of greater Melbourne. Project physiotherapists implemented both interventions at no cost to the participants. They were trained in the implementation of both the physiotherapy and placebo intervention by JM and were provided with a detailed instruction manual. All treatments were individual sessions lasting 30 to 45 minutes once weekly for four weeks, then fortnightly for eight weeks. During the follow up period there was no contact between the physiotherapist and the participants. The use of drugs and other treatments was recorded.

\section{Physiotherapy}

A standardised treatment was devised (JM). This included knee taping; exercises to retrain the quadriceps, hip, and back muscles; balance exercises; thoracic spine mobilisation; and soft tissue massage (table 1). Home exercises were to be done three times daily. Participants were provided with standardised home exercise and taping instruction sheets. Compliance with home exercises was monitored in a log book. 


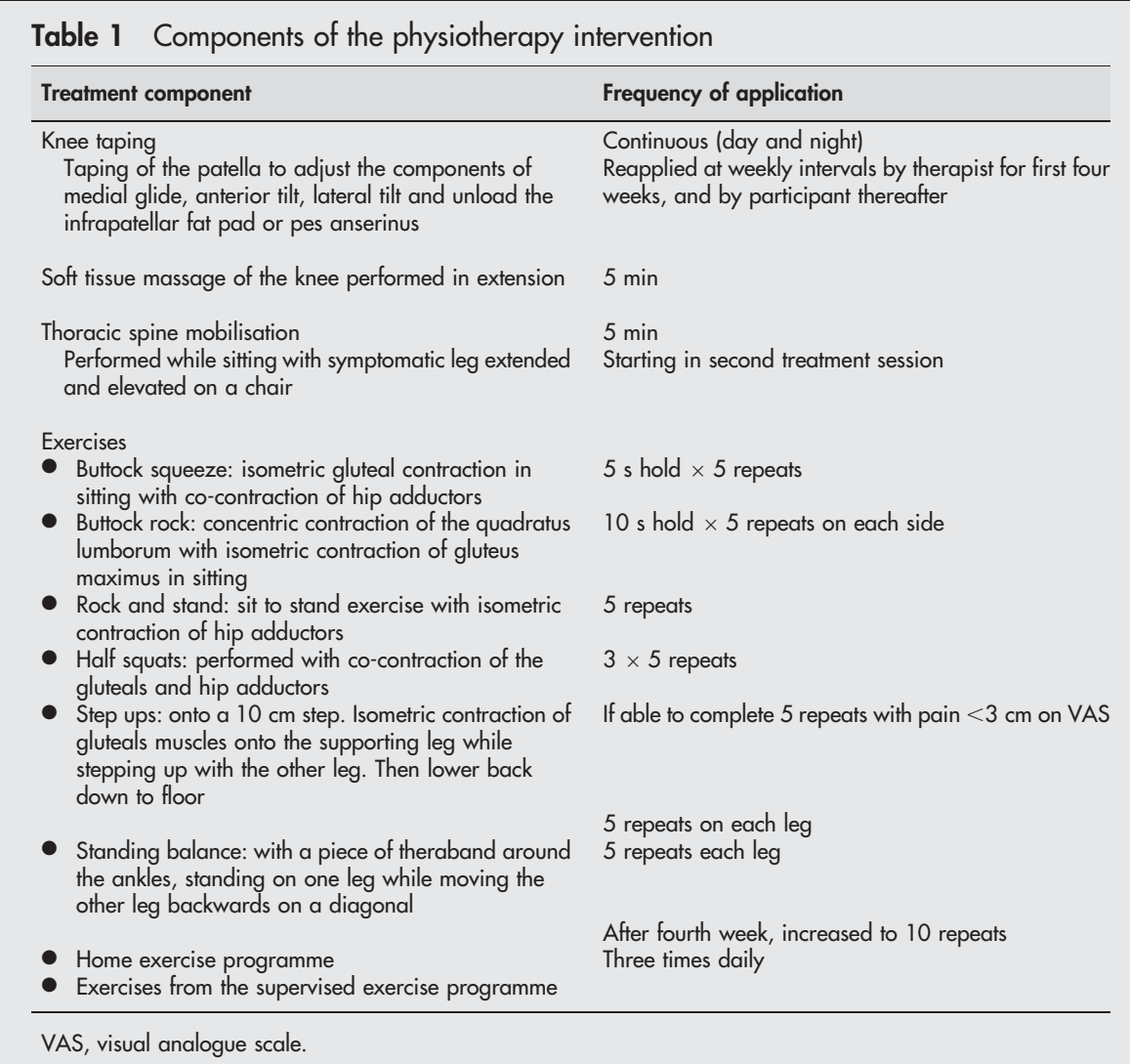

Knee taping consisted of non-rigid, hypoallergenic tape (Fixomull, Beiersdorf Australia, North Ryde) to provide skin protection, and rigid strapping tape (Beiersdorf Australia) for patellar adjustments, to be worn continuously ${ }^{12}$ (table 1). Appropriate self application of the tape once weekly (from week 5 onwards) was assessed by the treating therapist.

During the follow up, the physiotherapy group continued the daily home exercises and knee taping for alternate weeks for six weeks, then as needed thereafter. Tape use and exercise completion was recorded in a log book.

\section{Placebo}

Placebo treatment consisted of sham ultrasound (custom made, Metron, Australia) and light application of nontherapeutic gel (ultrasonic gel, Parker Laboratories Inc, New Jersey, USA and Hospital Skin Care Lotion, Smith \& Nephew, Clayton, Australia). ${ }^{7}$ The placebo participants did not receive any intervention during the follow up period.

\section{Masking}

A blinded examiner (BM) carried out all outcome assessments. Participant blinding was optimised by using a realistic placebo intervention and by ensuring participants did not attend for treatments or assessments concurrently. At trial completion, participants nominated which group they believed they had been allocated to. The data manager and statistician were unaware of treatment allocation until completion of analyses.

\section{Primary outcomes}

Pain on movement (over the past week) was measured by a visual analogue scale (VAS) ${ }^{13}$ numbered in $1 \mathrm{~cm}$ intervals. Patient global change in pain (since the start of the trial) was recorded on a five point Likert scale, ranging from 1 (much worse) to 5 (much better). Participants were classified as "improvers" using two methods: those who scored their global change in pain as 4 or 5; and those who reported a clinically significant change in pain $(\geqslant 1.75 \mathrm{~cm}$ on VAS).

\section{Secondary outcomes}

Questionnaires

Measures of pain, disability, and quality of life included the Western Ontario and McMaster Universities osteoarthritis index (WOMAC), ${ }^{14}$ the knee pain scale, ${ }^{15}$ an 11 point numerical VAS (average restriction of activity), the short form 36 item general health questionnaire (SF-36), ${ }^{16}$ and the assessment of quality of life (AQoL) index. ${ }^{17}$

\section{Step test}

The step test is a functional, dynamic test of standing balance. ${ }^{18}$ The number of times the foot can be placed up onto a $15 \mathrm{~cm}$ step and returned to the floor in 15 seconds while balancing on the symptomatic leg is recorded.

\section{Isometric quadriceps strength}

This was assessed at $60^{\circ}$ knee flexion in the sitting position using a KinCom dynamometer (Chattecx Corporation, Chattanooga, Tennessee, USA). A submaximal warm up was followed by three maximal $5 \mathrm{~s}$ contractions with a $15 \mathrm{~s}$ rest interval. The highest peak force of the three trials normalised for body weight was used.

\section{Sample size}

Pain reduction of $1.75 \mathrm{~cm}$ on VAS is recommended as the minimum clinically important difference in osteoarthritis trials. ${ }^{19}$ With 126 participants, the study had $90 \%$ power to detect a difference in pain reduction of $1.75 \mathrm{~cm}$ between treatment groups, assuming a standard deviation of $3.0 \mathrm{~cm}$ with a significance level of $5 \%$. Numbers were increased to 140 to allow for drop outs. 


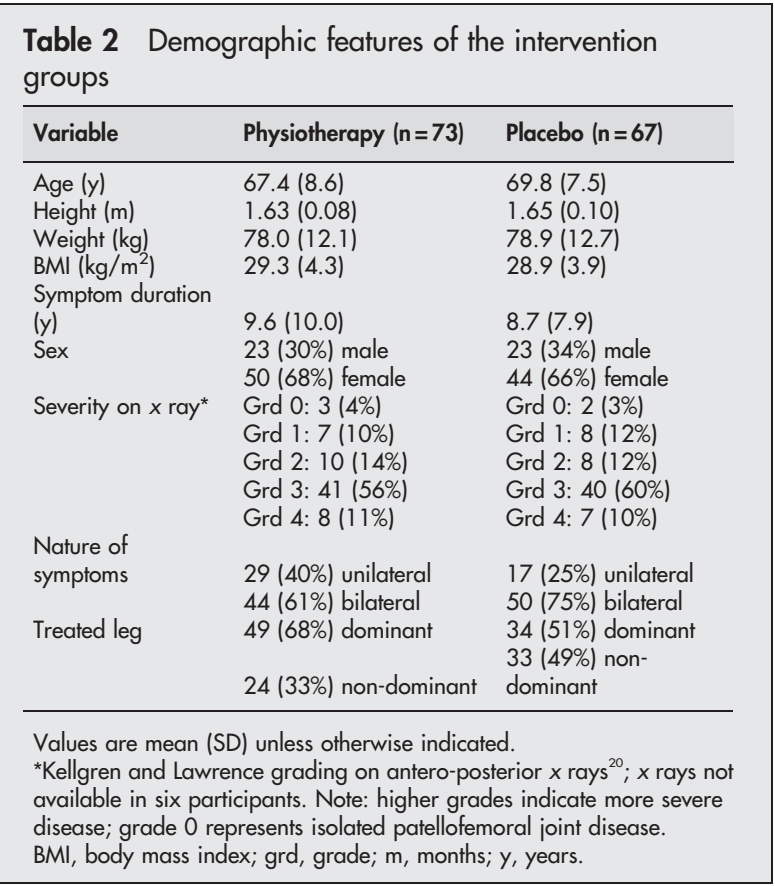

\section{Statistical analysis}

Statistical analyses, employing SPSS software, were undertaken on an intention to treat basis using a conservative method for replacing missing data. In the physiotherapy participants, missing data were replaced by the placebo group mean, and vice versa for missing placebo data, except for the global change outcome where missing data from participants in both groups were allocated a score of 3 ("no change"). In addition, a per protocol analysis was carried out to see if results differed. For each participant, change scores were calculated by subtracting the result of the final and follow up assessment from those at baseline. We calculated mean change scores within groups, and differences in change scores between groups, with $95 \%$ confidence intervals. Comparison of the numbers of "improvers" in each treatment group was done with $\chi^{2}$ tests.

\section{RESULTS}

Between May 2000 and May 2002, 650 volunteers were screened. Of these, 140 enrolled in the trial with 73 randomised to physiotherapy and 67 to placebo (fig 1). Groups were comparable at baseline (tables 2 and 3). The 12 week programme was completed by 124 participants (59 physiotherapy, 65 placebo) with 13 drop outs in the physiotherapy group (18\%) and two in the placebo group (3\%). Follow up was completed by 119 participants (85\%) (56 physiotherapy, 63 placebo). Those participants who dropped out did not differ at baseline from those who completed final measurements (except for height, where the completers were shorter).

\section{Change immediately following intervention Primary outcomes}

Both groups showed reduced pain on movement following intervention (table 3). Pain reduction averaged $42 \%$ with physiotherapy treatment and 38\% with placebo treatment. Seventy per cent of physiotherapy participants and $72 \%$ of placebo participants reported global improvement. A clinically relevant reduction in pain on VAS was reported by $53 \%$ of the physiotherapy group and $47 \%$ of the placebo group. The proportion of "improvers" with each intervention was similar (both $\mathrm{p}>0.05$ ).

\section{Secondary outcomes}

Both groups showed similar improvements on all secondary outcomes (table 3) except for quadriceps strength, where there was no change in either group.

\section{Change at follow up \\ Primary outcomes}

Movement pain (VAS) remained significantly lower at follow up than at baseline in both groups (table 3). A similar percentage of physiotherapy (59\%) and placebo (50\%) participants reported global improvement (from the start of the trial) $(p=0.309)$. Similar proportions of physiotherapy participants (58\%) and placebo participants (42\%) reported a clinically relevant reduction in pain on VAS $(p=0.069)$.

\section{Secondary outcomes}

With both interventions, most secondary measures remained improved from baseline (table 3 ). However, a significantly greater improvement in quality of life was evident in the physiotherapy group compared with the placebo group.

\section{Per protocol analysis}

When the primary outcomes were reanalysed using only the data from those who completed the trial, there was no difference between groups for pain reduction and global improvement at 12 weeks (both $\mathrm{p}>0.05$ ). At 24 weeks, movement pain remained significantly lower than at baseline in both groups. However, a greater proportion of physiotherapy participants $(77 \%)$ reported global improvement (from baseline) compared with placebo participants (49\%) $(\mathrm{p}=0.005)$ at this time. More physiotherapy participants $(66 \%)$ reported a clinically relevant reduction in pain on VAS than did placebo participants $(48 \%)(\mathrm{p}=0.027)$.

\section{Compliance, adverse events, and co-interventions}

Participants were compliant during the intervention period, with $95 \%$ of treatment sessions attended. During the intervention and follow up periods, $72 \%$ and $50 \%$ of home exercise sessions were completed, respectively. During follow up, $45 \%$ of the physiotherapy group taped their knee for a mean (SD) of 29 (13) days. Minor and short lived adverse events were reported during the interventions. In the physiotherapy group, these included minor skin irritation $(48 \%)$, increased pain with exercises $(22 \%)$, and pain with massage (1\%). In the placebo group, these included increased pain $(2 \%)$ and itchiness and pain with application of gel $(2 \%)$. At follow up, $8 \%$ of physiotherapy participants reported knee/back pain associated with home exercises, while $1 \%$ reported skin problems with taping. Drug use was similar between the physiotherapy and placebo groups over the treatment period (analgesics, 23\% v 21\%; non-steroidal antiinflammatory drugs, $22 \%$ v 24\%; glucosamine, $3 \% \vee 6 \%$ ). No other co-interventions were reported by the physiotherapy group, while $1 \%$ of placebo participants reported attending a podiatrist and wearing a magnetic bandage.

\section{Success of blinding}

Sixty seven per cent of the placebo group remained blinded to their group allocation. The assessor was unblinded for six $(10 \%)$ and two $(3 \%)$ of the physiotherapy and placebo final assessments, respectively, and for nine (16\%) and two (3\%) of the physiotherapy and placebo follow up assessments.

\section{DISCUSSION}

We conducted a randomised, double blind, placebo controlled trial of a novel multimodal physiotherapy package in a large cohort of community volunteers with symptomatic knee osteoarthritis. We showed significantly improved pain and function with both physiotherapy and placebo interventions. 
Table 3 Primary and secondary outcome scores over time according to group allocation using intention to treat analysis

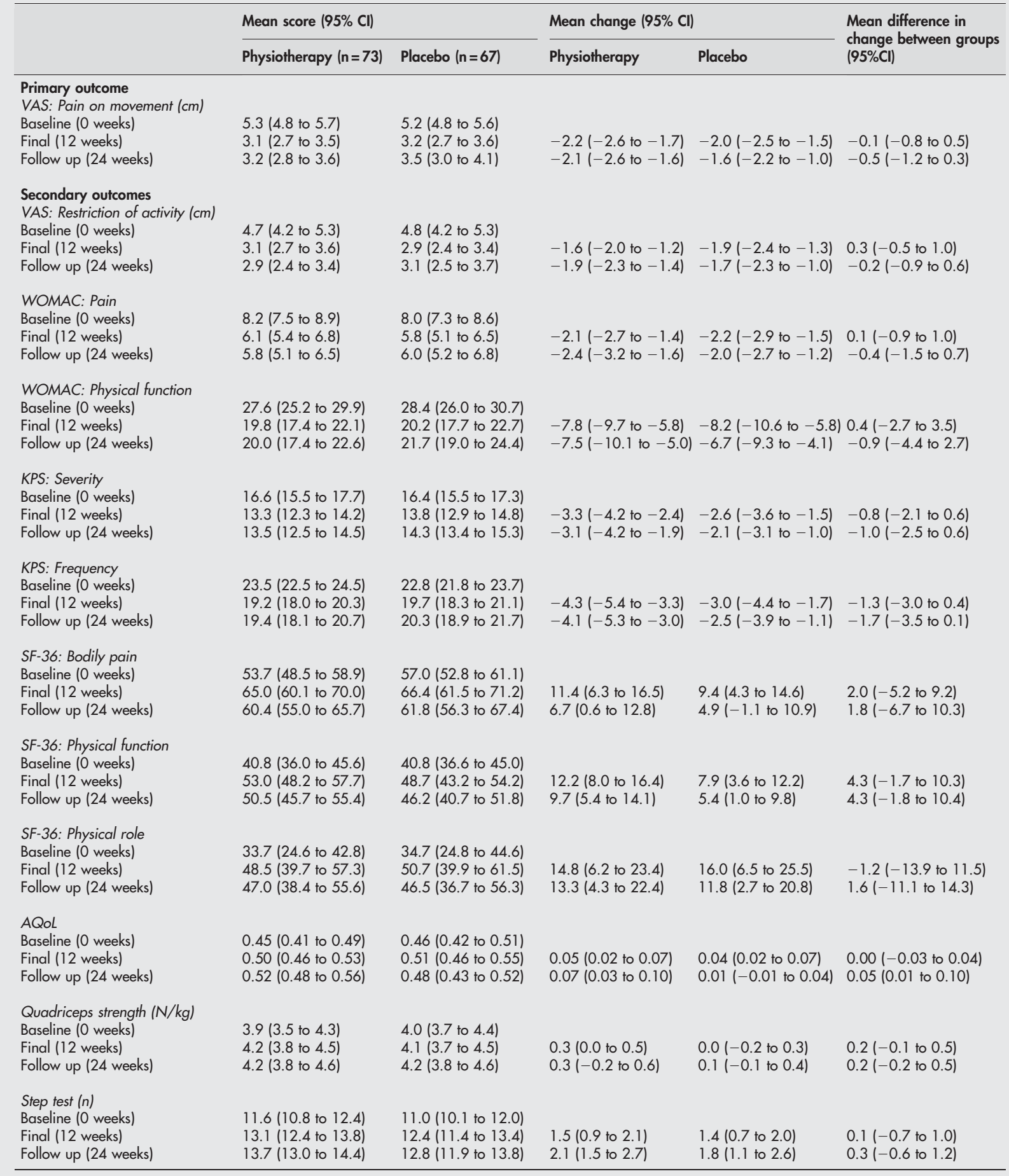

NOTE: there were no significant differences in baseline scores across intervention groups.

$\mathrm{AQoL}$, assessment of quality of life index (ranging from -0.04 to 1.0, with higher scores indicating greater quality of life; Cl, confidence interval; KPS, knee pain scale (severity subscale ranging from 0 to 36 points and the frequency subscale ranging from 0 to 30 points, with higher scores indicating more severe or more frequent pain); SF-36, Medical Outcomes Study 36 item short form (ranging from 0 to 100 points, with higher scores indicating better quality of life); VAS, visual analogue scale (ranging from $0=$ no pain to $10=$ worst pain possible); WOMAC, Western Ontario and McMaster Universities osteoarthritis index (pain scores range from 0 to 20 points and physical function scores from 0 to 68 , with higher scores indicating more severe pain/physical function).

Pain reduction was similar in both groups, and of a clinically relevant magnitude in around half the participants. This suggests that the physiotherapy package investigated in this trial offered no greater benefits than regular contact with a therapist.

In our study, the improvements seen immediately following intervention could reflect the natural history of the disease. Symptoms associated with chronic conditions such as knee osteoarthritis typically fluctuate over time, ${ }^{21}$ with patients often seeking medical care or enrolling in research when the symptoms are at their worst. Thus the next change in symptoms is likely to be an improvement. A tendency for extreme symptoms at baseline to return towards a more typical state at final assessment is also known as regression 
toward the mean. ${ }^{22}$ While we did not include a third "no treatment" arm, we believe that natural history is an unlikely explanation. Another trial conducted by our group using identical recruitment and selection criteria and outcome measures showed no change in pain and physical function over six weeks in the participants receiving no treatment. ${ }^{12}$

A possible explanation for our results is non-specific treatment effects, often known as placebo effects. These placebo effects may be attributed to the attention, interest, and concern displayed by the investigator or physiotherapist, patient expectations of treatment effects, expense and impressiveness of the intervention, characteristics of the healing setting, or the intense monitoring associated with a research trial. ${ }^{23}{ }^{24}$ Placebo effects are common in individuals with knee osteoarthritis, and have been demonstrated with a range of sham treatments including intra-articular saline injection, ${ }^{25}$ simulated arthroscopic procedures, ${ }^{9}$ and placebo glucosamine sulphate. ${ }^{10}$ In these studies, improvements with sham interventions ranged from $16 \%$ to $40 \%$. Our finding of a $38 \%$ reduction in pain with placebo is consistent with these studies. A meta-analysis of placebo controlled clinical trials ${ }^{26}$ has shown that placebos have their greatest effect on continuous subjective outcomes and in the treatment of pain. Given that psychological variables such as anxiety, helplessness, and pain coping have been correlated with pain and disability in knee osteoarthritis, ${ }^{27} 28$ and our successful blinding of the participants, it is not surprising that such a strong placebo response was seen in our trial. Furthermore, it must be noted that our placebo treatment involved physical contact with the skin. It is feasible that this had direct effects on the knee, as touch can reduce pain and disability in knee osteoarthritis. ${ }^{29}$

Another likely cause of the non-significant difference between groups following intervention may relate to our use of a novel programme with an emphasis on motor control and function, particularly around the hip and pelvis, rather than on lower limb strengthening and aerobic fitness. The exercise component was similar to that used by Quilty et al, ${ }^{8}$ who, like us, showed little increase in quadriceps strength and no overall effect of the physiotherapy programme. These non-significant findings may highlight the importance of muscle strengthening exercises for knee osteoarthritis, particularly given the significant reductions in pain following resistance training in other studies. ${ }^{30} 31$

Taping was a component of our programme and has been shown by us $^{12}$ and others ${ }^{30}$ to be effective in this patient population when applied by a trained therapist. In our present study and that of Quilty et al, ${ }^{8}$ the taping was generally carried out by the patients themselves. The nonsignificant effects in both studies brings into question the effectiveness of patient applied tape in knee osteoarthritis. Future studies should investigate the efficacy of self versus therapist application of tape, as this will influence the clinical applicability of this form of treatment.

Our physiotherapy programme was standardised, with limited scope for exercise progression; given the heterogeneity of our sample, it is possible that it was less effective for some participants than for others. This may have contributed to our lack of a significant between group differences. It is possible that greater improvements may have been noted with individualised physiotherapy treatment. This is certainly feasible given evidence suggesting that knee osteoarthritis subgroups respond differently to treatment interventions. ${ }^{6}$

Before our study, only three randomised controlled trials of multimodal physiotherapy programmes for knee osteoarthritis had been conducted..$^{6-8}$ Of these, only Quilty et $a l^{8}$ used a comparable physiotherapy programme to ours but in a cohort with predominant patellofemoral osteoarthritis. As in our study, the investigators found no difference between groups in pain or function. In the only other placebo controlled trial of physiotherapy, Deyle et al found significant benefits with a four week programme. The different results compared with ours may reflect a more homogeneous osteoarthritis group and an individually tailored treatment programme with a greater emphasis on quadriceps strengthening. In the third trial, physiotherapy delivered individually or in a group exercise class format resulted in greater improvement than in a no treatment control group. ${ }^{6}$ No placebo group or controlled follow up was available in this study to compare with ours.

We used a conservative method to allocate values for missing data in our intention to treat analysis, and the effect of the drop outs is unknown. However, when reanalysing the data on a per protocol method, similar results were found at 12 weeks. At 24 weeks, the physiotherapy group reported greater global improvement than the placebo group. These results suggest that placebo effects are less likely to be maintained once contact with the therapist has ceased.

Future research should focus on determining which factors influence the treatment responsiveness of patients with knee osteoarthritis. As knee osteoarthritis is a heterogeneous condition, it is possible that subgroups of patients may respond differently to different modes of physiotherapy. This will allow the development of targeted physiotherapy programmes for particular subgroups. Economic analyses should also be incorporated into research designs to allow clinicians and health care providers to determine the most cost-effective forms of physiotherapy treatment.

\section{ACKNOWLEDGEMENTS}

This work was funded by the National Health and Medical Research Council (grant No 114277). KC is supported by a NHMRC postdoctoral fellowship. We thank Dr Mike Smith for evaluating the radiographs and Helen Archer, Jane Burman, Emma Colson, Lena Fiddelaers, Peter Kent, Jean Leaf, Janet Pullar, Christine Roberts, Ann Ryan, and Simon Wilson for administering the interventions.

\section{Authors' affiliations}

K L Bennell, R S Hinman, B R Metcalf, J McConnell, K M Crossley,

Centre for Health, Exercise and Sports Medicine, School of Physiotherapy, University of Melbourne, Parkville, Victoria, Australia R Buchbinder, Monash Department of Clinical Epidemiology, Cabrini Hospital and Department of Epidemiology and Preventive Medicine, Monash University, Melbourne, Australia

G McColl, Department of Medicine, Royal Melbourne Hospital, University of Melbourne

S Green, Monash Institute of Health Services Research, Monash University

\section{REFERENCES}

1 Felson DT, Naimark A, Anderson J, Kazis L, Castelli W, Meenan RF. The prevalence of knee osteoarthritis in the elderly. The Framingham Osteoarthritis Study. Arthritis Rheum 1987;30:914-18.

2 Arthritis Foundation of Australia. The prevalence, cost and disease burden of arthritis in Australia. Canberra: Access Economics, 2001.

3 Hamerman D. Clinical implications of osteoarthritis and aging. Ann Rheum Dis 1995; 54:82-5.

4 Jordan K, Arden N, Doherty M, Bannwarth B, Bijlsma J, Dieppe P, et al. EULAR recommendations 2003: an evidence based approach to the management of knee osteoarthritis. Report of a task force of the Standing Committee for International Clinical Studies Including Therapeutic Trials (ESCISIT). Ann Rheum Dis 2003;62:1145-55.

5 American College of Rheumatology. Subcommittee on Osteoarthritis Guidelines. Recommendations for the medical management of osteoarthritis of the hip and knee, 2000 update. Arthritis Rheum 2000;43:1905-15.

6 Fransen M, Crosbie J, Edmonds J. Physical therapy is effective for patients with osteoarthritis of the knee: a randomized controlled trial. J Rheumatol $2001 ; 28: 156-64$.

7 Deyle GD, Henderson NE, Matekel RL, Ryder MG, Garber MB, Allison SC. Effectiveness of manual physical therapy and exercise in osteoarthritis of the knee. A randomized, controlled trial. Ann Internal Med 2000;132:173-81.

8 Quilty B, Tucker M, Campbell R, Dieppe P. Physiotherapy, including quadriceps exercises and patellar taping, for knee osteoarthritis with 
predominant patello-femoral joint involvement: Randomized controlled trial. $J$ Rheumatol 2003;30:1311-17.

9 Moseley BJ, O'Malley K, Petersen NJ, Menke TJ, Brody BA, Kuykendall DH, et al. A controlled trial of arthroscopic surgery for osteoarthritis of the knee. N Engl J Med 2002;347:81-8.

10 Hughes R, Carr A. A randomized, double-blind, placebo-controlled trial of glucosamine sulphate as an analgesic in osteoarthritis of the knee. Rheumatology 2002;41:279-84.

11 Altman R, Asch E, Bloch D, Bole G, Borenstein D, Brandt K, et al. Development of criteria for the classification and reporting of osteoarthritis. Classification of osteoarthritis of the knee. Arthritis Rheum 1986;29:1039-49.

12 Hinman R, Crossley K, McConnell J, Bennell K. Efficacy of knee tape in the management of knee osteoarthritis: A blinded randomised controlled trial. BMJ 2003;327:135-8.

13 Huskisson EC. Visual analogue scales. In: Melzack R, eds. Pain measurement and assessment. New York: Raven Press, 1983:33-7.

14 Bellamy N, Buchanan WW, Goldsmith CH, Campbell J, Stitt LW. Validation study of WOMAC: a health status instrument for measuring clinically important patient relevant outcomes to antirheumatic drug therapy in patients with osteoarthritis of the hip or knee. J Rheumatol 1988;15:1833-40.

15 Rejeski WJ, Ettinger WHJ, Shumaker S, Heuser MD, James P, Monu J, et al. The evaluation of pain in patients with knee osteoarthritis: the knee pain scale. J Rheumatol 1995;22:1124-9.

16 Ware J, Sherbourne C. The MOS 36-item short-form health survey (SF-36). Med Care 1992;30:473-83.

17 Hawthorne G, Richardson J, Day N. A comparison of the assessment of quality of life (AQoL) with four other generic utility instruments. Ann Med 2001;33:358-70.

18 Hill K, Bernhardt J, McGann AM, Maltese D, Berkovits D. A new test of dynamic standing balance for stroke patients: reliability, validity and comparison with healthy elderly. Physiother Canada 1996;48:257-62.

19 Bellamy N, Carette S, Ford P, Kean W, le Riche N, Lussier A, et al. Osteoarthritis antirheumatic drug trials. III. Setting the delta for clinical trials: results of a consensus development (delphi) exercise, J Rheumatol 1992; 19:451-7.

20 Kellgren JH, Lawrence JS. Radiological assessment of osteoarthrosis. Ann Rheum Dis 1957; 16:494-501.

21 Bellamy N, Sothern RB, Campbell J. Rhythmic variations in pain perception in osteoarthrits of the knee. J Rheumatol 1990;17:364-72.

22 Whitney CW, Von Korff M. Regression to the mean in treated versus untreated chronic pain. Pain 1992;50:281-5.

23 Kaptchuk CJ. The placebo effect in alternative medicine: can the performance of a healing ritual have clinical significance? Ann Intern Med 2002;136:817-25.

24 Turner J, Deyo R, Loesser J, Von Korff M, Fordyce W. The importance of placebo effects in pain treatment and research. JAMA 1994;271:1609-14.

25 Hassan B, Doherty S, Mockett S, Doherty M. Effect of pain reduction on postural sway, proprioception, and quadriceps strength in subjects with knee osteoarthritis. Ann Rheum Dis 2002;61:422-8.

26 Hrobjartsson A, Gotzsche P. Is the placebo powerless? An analysis of clinical trials comparing placebo with no treatment. N Engl J Med 2001;344:1594-602

27 Creamer P, Lethbridge-Cejku M, Hochberg MC. Determinants of pain severity in knee osteoarthritis: effect of demographic and psychosocial variables using 3 pain measures. J Rheumatol 1999;26:1785-92.

28 Creamer $\mathbf{P}$, Lethbridge-Cejku M, Hochberg MC. Factors associated with functional impairment in symptomatic knee osteoarthritis. Rheumatology 2000;39:490-6.

29 Gordon A, Merenstein JH, Damico F, Hudgens D. The effects of therapeutic touch on patients with osteoarthritis of the knee. J Family Prac 1998:47:271-7.

30 Thomas KS, Muir KR, Doherty M, Jones AC, O'Reilly SC, Bassey EJ. Home based exercise programme for knee pain and knee osteoarthritis: randomised controlled trial. BMJ 2002;325:752-6.

31 Topp R, Woolley S, Hornyak J, Khuder S, Kahaleh B. The effect of dynamic versus isometric resistance training on pain and functioning among adults with osteoarthritis of the knee. Arch Phys Med Rehabil 2002;83:1187-95. 